\title{
Sturdier DNA Nanotubes via Ligation
}

Patrick O’Neill, ${ }^{\dagger}$ Paul W. K. Rothemund, ${ }^{\ddagger}$ Ashish Kumar ${ }^{\dagger}{ }^{\text {and }}$ D. K. Fygenson ${ }^{\star, t, \S}$

Department of Physics, University of California, Santa Barbara, California 93106, Department of Computer Science and Computational and Neural Systems, California Institute of Technology, Pasadena, California 91125, and Biomolecular Science \& Engineering Program, University of California, Santa Barbara, California 93106

Received February 15, 2006; Revised Manuscript Received May 19, 2006

\begin{abstract}
DNA nanotubes are crystalline self-assemblies of DNA tiles $\sim 10 \mathrm{~nm}$ in diameter that readily grow tens of micrometers in length. Easy assembly, programmability, and stiffness make them interesting for many applications, but DNA nanotubes begin to melt at temperatures below $40{ }^{\circ} \mathrm{C}$, break open when deposited on mica or scanned by AFM, and disintegrate in deionized water. These weaknesses can be traced to the presence of discontinuities in the phosphate backbone, called nicks. The nanotubes studied here have five nicks, one in the core of a tile and one at each corner. We report the successful ligation of all four corner nicks by T4 DNA ligase. Although ligation does not change the nanotubes' stiffness, ligated nanotubes withstand temperatures over $70^{\circ} \mathrm{C}$, resist breaking during AFM, and are stable in pure water for over a month. Ligated DNA nanotubes are thus physically and chemically sturdy enough to withstand the manipulations necessary for many technological applications.
\end{abstract}

Made entirely of DNA, but hundreds of times stiffer than ordinary double-stranded DNA (dsDNA), DNA nanotubes combine the binding specificity of nucleic acids with a rigid, linear structure. ${ }^{1-3}$ This combination of structural and biochemical properties makes DNA nanotubes especially interesting for applications. For example, they might be metallized or functionalized by surface attachment of biomolecules or nanoparticles and so serve as interconnects in self-assembled networks. ${ }^{4-8}$ Alternatively, DNA nanotubes might be used, like actin filaments, ${ }^{9}$ as mechanical magnifiers of the nanoscopic motion of biomolecules, but with greater chemical stability and versatility. However, the practical realization of these and many other applications will require DNA nanotubes that withstand considerable mechanical and chemical manipulation. Therefore, to enhance the technological relevance of DNA nanotubes, we sought to increase their stability via ligation.

Ligation is commonly used to establish the topology of programmed DNA nanostructures. ${ }^{10-13}$ However, its effectiveness within a two-dimensional DNA tile array has never been well characterized. ${ }^{14}$ Crystal structures of ligase enzymes suggest that ligation requires the enzyme to access a large portion of the double helical surface. ${ }^{15}$ One might therefore expect ligation among the potentially close-packed double helices of the nanotube tile lattice to be particularly inhibited.

\footnotetext{
* Corresponding author. E-mail: deborah@physics.ucsb.edu.

† Department of Physics, University of California, Santa Barbara.

$\div$ California Institute of Technology.

$\S$ Biomolecular Science \& Engineering Program, University of California, Santa Barbara.
}

In this paper, we report that DNA nanotubes are viable substrates for T4 DNA ligase, quantify the extent of ligation at each of the five nicks, and show that ligated nanotubes are significantly more stable to practical manipulation than unligated nanotubes.

The DNA nanotubes that we use self-assemble from a single DAE-E type ${ }^{16}$ tile (Figure 1). The tile is made of five synthetic DNA oligomers that hybridize into a rigid rectangular core with a single-stranded five-base overhang (sticky end) at each corner. The core consists of two double helices joined at two four-way junctions (crossovers). Diagonally opposite corners present complimentary sticky ends. When these hybridize, the tiles form a 2D lattice, but the lattice is not flat. The intrinsic twist of dsDNA and the number of base pairs (bp) between crossovers in neighboring tiles constrain them to join at $\mathrm{a} \sim 150^{\circ}$ angle about their common double helix, imparting an intrinsic curvature to the lattice that favors closure with a diameter of $\sim 10 \mathrm{~nm}^{3}$.

Each sticky end junction, where DNA strands from adjoining tiles meet upon hybridization, is a short (5 bp) segment of dsDNA flanked by a pair of nicks. Nicks are gaps in an otherwise continuous sugar-phosphate backbone. They are therefore weak points at which tiles, or perhaps individual strands, dissociate in response to changes in temperature, electrostatic environment, and solvent conditions. There is also a nick in the center of each tile, where the two ends of strand no. 3 meet upon tile formation. Strand no. 3 is referred to as the core strand, and the associated nick is called the core nick. The other four nicks are called corner nicks. 
(A)

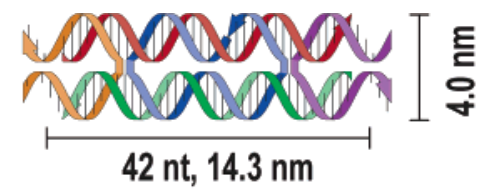

(B)

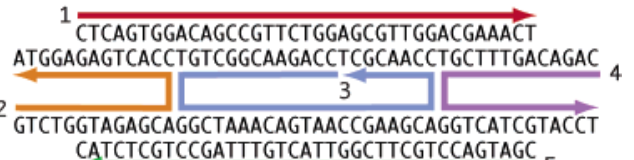

(C)

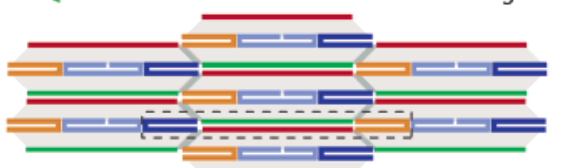

(D)

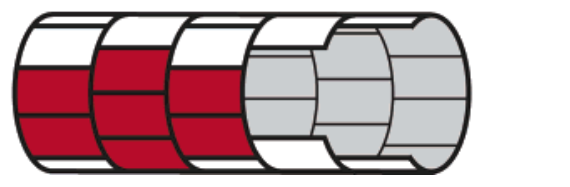

Figure 1. Schematics of the DNA tile and nanotube used. (A) Double helical representation of a single tile. Arrowheads mark the $3^{\prime}$ end of each strand. (B) Sequence and numbering scheme for each strand in the tile. (C) Small portion of lattice, consisting of seven tiles. The dashed box frames a region where crossovers six helical turns apart connect neighboring helices. Regions where neighboring helices are connected by crossovers only two helical turns apart are found at the location of each strand no. 3. (D) Short nanotube segment with a red region corresponding to the seventile array depicted in $\mathrm{C}$.

T4 DNA ligase is an ATP-driven enzyme that seals nicks. More precisely, it catalyzes the formation of a covalent phosphodiester bond between the $5^{\prime}$ phosphate of one DNA strand and the $3^{\prime}$ hydroxyl of another. The terminal 5' phosphate is typically absent in synthetic DNA, but can be added chemically, or by another ATP-driven reaction using the enzyme DNA kinase. One may therefore control which nicks in the lattice can be ligated by selectively phosphorylating only a subset of the strands comprising the tile. We used this control to study the extent and consequence of ligation on DNA nanotubes. ${ }^{17}$

In our nanotube lattice, strand no. 1 ligates to strand no. 2, strand no. 2 ligates to strand no. 5 , strand no. 5 ligates to strand no. 4 , and strand no. 4 ligates to the original strand no. 1. Complete ligation would thus result in a meshwork of concatenated loops of ssDNA. ${ }^{18}$

DNA nanotubes in which one, two (strand nos. 2 and 4), three (strand nos. 1, 2, and 4), four (strand nos. 1, 2, 4, and 5 ), or all five strands were chemically phosphorylated (on the $5^{\prime}$ end) are referred to herein as one-point, two-corner, three-corner, four-corner, and five-point phosphorylated tubes, respectively (Figure 1B). All five strands were mixed at $1.8 \mu \mathrm{M}$ (each strand) in a buffer consisting of $30 \mathrm{mM}$ tris- $\mathrm{HCl}$ (pH 7.8), $10 \mathrm{mM}$ DTT, $1 \mathrm{mM} \mathrm{ATP}$, and $10 \mathrm{mM}$ $\mathrm{MgCl}_{2} \cdot{ }^{19}$ Thin-walled $200 \mu \mathrm{L}$ PCR tubes containing $30 \mu \mathrm{L}$ of the strand mixture were submerged in a $2 \mathrm{~L}$ beaker of 90 ${ }^{\circ} \mathrm{C}$ water, which was placed in a Styrofoam box and left to cool to room temperature over $48 \mathrm{~h}$. This procedure (the anneal) results in a high density of nanotubes, many over $20 \mu \mathrm{m}$ long, as verified by fluorescence microscopy (see below).

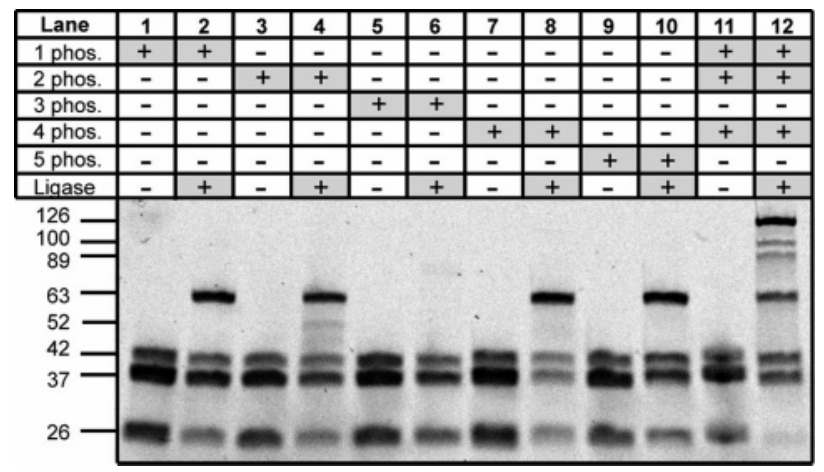

Figure 2. Denaturing polyacrylamide gel comparing strands from ligated and unligated DNA nanotubes with different strands phosphorylated. Lanes 1-10: uppermost bands correspond to 63 base long ligation product (either no. 14, 45, 52, or 21) as determined by extrapolation based on the known lengths of strands in the lower (unligated) bands (nos. 2 and 4: 26 bases, nos. 1 and 5: 37 bases, no. 3: 42 bases). No slowly migrating band corresponding to a cyclized strand no. 3 is detected (lane 6). Note that each corner ligation involves only one of the two 26 base strands (lanes 2, 4, 8, and 10). Lanes 11 and 12: uppermost band corresponds to a 126 base strand, generated when three contiguous nicks are ligated to form one large strand (no. 5412). Strands of 100 bases (no. 521) and 89 bases (no. 214) are generated when only two contiguous nicks are ligated.

For ligation, T4 DNA ligase (Fisher Cat. No. BP3210-1), ATP, and DTT were added to the annealed nanotube solution. Final concentrations in the ligation mixture were as follows: $[\mathrm{DNA}]=1.2 \mu \mathrm{M}$ (each strand), [ligase] $=0.5$ units/ $\mu \mathrm{L}, 0.9 \mathrm{mM}<[\mathrm{ATP}]<1.6 \mathrm{mM}$, and $8.6 \mathrm{mM}<[\mathrm{DTT}]<$ $15.3 \mathrm{mM}$. The upper (lower) bounds assume that none (all) of the ATP or DTT initially present was degraded during the anneal. Ligation mixtures were incubated at room temperature for at least 1 day prior to all experiments.

To assess the extent of ligation at each of the different nicks, we performed denaturing gel electrophoresis after ligating nanotubes in which only one of the five strands was $5^{\prime}$ phosphorylated (Figure 2). Bands were quantitated based on the fluorescence of the intercalating dye Sybr gold (Molecular Probes Cat. No. S11494). Comparing strands from nanotubes of identical composition, before and after exposure to ligase, we find that ligation takes place at each of the four corner nicks (Figure 2, lanes 2, 4, 8, and 10), but not at the nick in the tile core (Figure 2, lane 6). ${ }^{20}$

Accessibility of the corner nicks, and inaccessibility of the core nick, to ligase can be explained by the spacing between crossovers in the DNA lattice. Each corner nick lies in a region of helix that is linked to neighboring helices by crossovers that are six helical turns apart. This region may flex radially outward or inward, or neighboring helices may bow laterally, allowing ligase to access a large portion of the double-helical surface. The latter suggestion is supported by high-resolution AFM images of tile lattices on mica that show relatively wide separations (with an apparent width of 2-3 nanometers) in these regions. ${ }^{3}$

The core nicks, by contrast, each lie on a region of helix connected to one of its neighbors by crossovers only two helical turns apart. High-resolution AFM shows the separation between helices in this region to be $<0.5 \mathrm{~nm}$. The core 
nick faces this small intratile gap. Accordingly, we found that the core nick is inaccessible to ligase even in isolated tiles. Tiles with $5^{\prime}$-phosphorylated strand no. 3 and four noncomplimentary sticky ends were exposed to ligase, and the resulting strands were separated on a denaturing gel (not shown). ${ }^{21}$ Again, no band corresponding to cyclized strand no. 3 was detected. We suspect the core nick would be ligated if it faced the larger intertile gap. Ligation of such outwardfacing core nicks has been demonstrated, albeit not in a twodimensional tile lattice. ${ }^{13}$

Quantitative interpretation of gel band intensities is complicated by the fact that Sybr gold is three times more fluorescent when bound to dsDNA as compared to ssDNA. ${ }^{22}$ We must therefore consider the possibility that the ligation product is not completely denatured. Assuming that the ligation product has either adopted its lowest energy secondary structure ${ }^{23,24}$ or remained entirely unhybridized, we place conservative lower and upper bounds on the extent of ligation at each corner nick: $52-85 \%, 46-74 \%, 51-82 \%$, and $54-$ $82 \%$ for nicks at the $5^{\prime}$ ends of strand nos. 1, 2, 4, and 5, respectively. (See the Supporting Information for details of the calculations.) A similar analysis of the ligation product of three-corner phosphorylated nanotubes, assuming ligation occurs with equal probability at each nick, places the extent of ligation between $68 \%$ and $77 \%$ per nick.

The consistency of these values suggests that corner nicks are ligated independently. Interestingly, there is no indication that ligase distinguishes between corner nicks on the interior (at the $5^{\prime}$ ends of strand nos. 1 and 5) or exterior (at the $5^{\prime}$ ends of strand nos. 2 and 4) face of a nanotube. (See the Supporting Information of ref 1 for 3D graphics depicting all strands in the context of a nanotube.) Clearly, not all sticky ends were ligated. For example, ligation of threecorner phosphorylated nanotubes yields one-corner and twocorner ligation products as well (Figure 2, lane 12). Although this may reflect finite reaction conditions or incomplete phosphorylation, it may also be a result of defects in the nanotube lattice that leave some sticky ends unhybridized. Thus, if care is taken to ensure that the ligation reaction goes to completion, the extent of ligation might be used as a simple assay for the density of defects.

Given the high proportion of ligated corner nicks, it is reasonable to expect qualitative changes in nanotube properties. We looked for changes in thermal and mechanical stability, stiffness, and buffer compatibility upon ligation.

A significant increase in thermal stability is evident by fluorescence microscopy (Figure 3, see the Supporting Information for experimental details). Fluorescence comes from an organic dye (FAM) covalently attached to the $5^{\prime}$ end of strand no. 3. Four solutions of nanotubes, differing only in the number of $5^{\prime}$ phosphorylated strands per tile, were exposed to ligase. They were then held at a fixed temperature for $30 \mathrm{~min}$ and imaged in fluorescence. The images are qualitatively divided into three groups: normal (top row), deformed (middle row), and melted (bottom row). For the temperatures sampled, only normal and melted tubes were seen for one-corner and three-corner ligated tubes. Each additional ligated tile corner enhanced the thermal stability

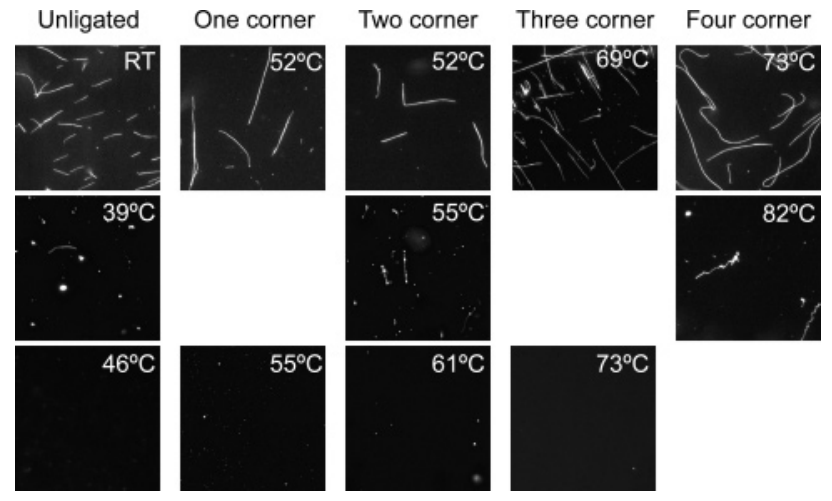

Figure 3. Fluorescent micrographs showing the increase in thermal stability as the number of ligation sites is increased. All of the nanotubes have been exposed to ligase, they differ only in the number of $5^{\prime}$ phosphorylated strands per tile. Phosphorylated strands: One-corner (strand no. 1), two-corner (strand nos. 2 and 4), three-corner (strand nos. 1, 2, and 4), four-corner (strand nos. 1, 2, 4, and 5). Top row: images from the highest temperatures sampled at which heated tubes were indistinguishable from unheated tubes. Middle row: images from intermediate temperatures at which tubes were deformed but not completely melted. For one-corner and three-corner ligated tubes, we did not find any such intermediate stability at the temperatures sampled. Bottom row: images from the lowest temperatures sampled at which tubes were completely melted. Images are $50 \mu \mathrm{m}$ on a side.

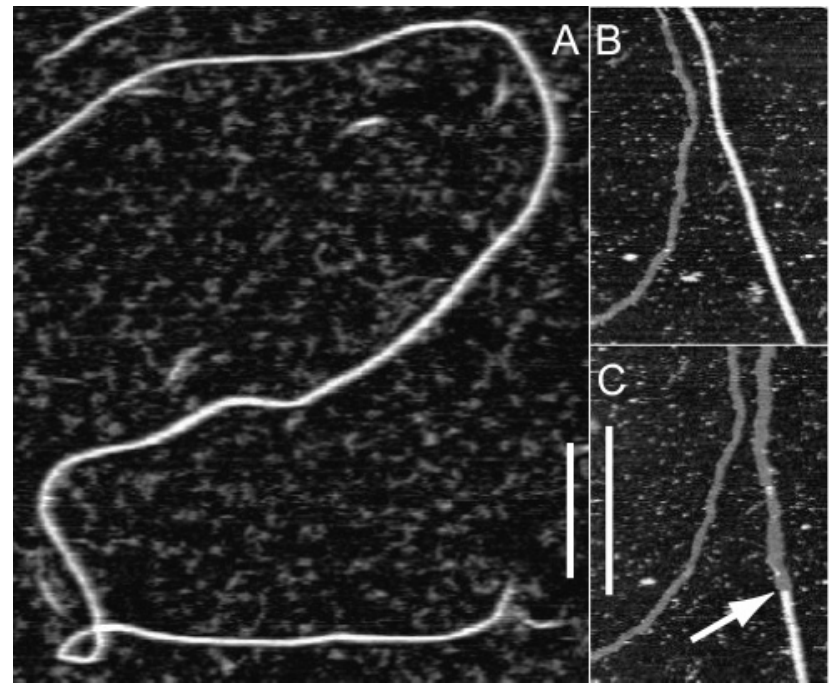

Figure 4. Atomic force micrographs of DNA nanotubes deposited on mica and imaged in tapping mode under TAE/Mg $1 \mathrm{X}$ buffer. A ligated nanotube (A) retains its streaky appearance and $\sim 5 \mathrm{~nm}$ thickness for the entire time of imaging $(\sim 1 \mathrm{~h})$. An unligated nanotube, initially closed (B, right), looks similar, but opens (C, right) into a $\sim 2$-nm-thick tile lattice within minutes of deposition. The displacement of tubes at the top of image (B) with respect to image (C) is an AFM artifact. Images b and c are adapted from ref 3. Scale bar: $500 \mathrm{~nm}$.

of the nanotubes (left to right). At the two extremes, unligated (nonphosphorylated) tubes melt completely by $46{ }^{\circ} \mathrm{C}$, whereas four-corner ligated tubes appear unaffected at temperatures up to $73{ }^{\circ} \mathrm{C}$.

Increased mechanical stability is evident by atomic force microscopy under buffer (Figure 4). After settling on the mica substrate, unligated nanotubes usually open within minutes, revealing the tile lattice. ${ }^{1,3}$ Under identical condi- 


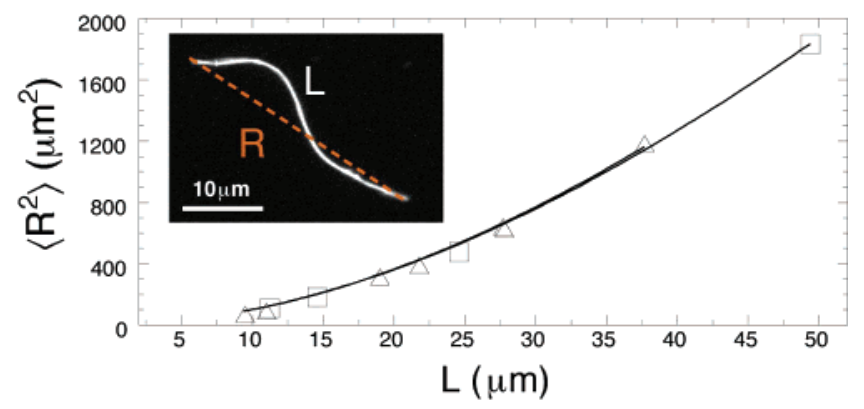

Figure 5. Average squared end-to-end distance $\left\langle R^{2}\right\rangle$ plotted against contour length $L$ (see inset) for ligated $(\triangle)$ and unligated $(\square)$ nanotubes. Lines are least-squares fits to the data using the 2D Kratky-Porod model. ${ }^{25}$ The resulting measurement of persistence length for ligated $(28.6 \pm 2.8 \mu \mathrm{m})$ and unligated $(26.2 \pm 1.4 \mu \mathrm{m})$ nanotubes is the same within error.

tions, four-corner ligated nanotubes remain intact for at least an hour. Not even aggressive imaging at high force was able to tear open the ligated tubes.

This enhanced mechanical stability raises the question of whether ligation changes the overall stiffness of a nanotube. We measured the stiffness of ligated and unligated nanotubes by observing thermally excited bending fluctuations of freefloating nanotubes confined to 2D (see the Supporting Information). The correlation between a given tube's average squared end-to-end distance, $\left\langle R^{2}\right\rangle$, and its contour length, $L$, relates to its stiffness, in terms of the persistence length, $p$, according to the wormlike chain model: ${ }^{25}$

$$
\left\langle R^{2}\right\rangle=4 p L\left[1-\frac{2 p}{L}\left(1-\mathrm{e}^{-L / 2 p}\right)\right]
$$

A plot of $\left\langle R^{2}\right\rangle$ versus $L$ for ligated and unligated nanotubes fits well to this function with $p$ as a free parameter (Figure 5). The resulting persistence lengths of ligated (28.6 \pm 2.8 $\mu \mathrm{m})$ and unligated $(26.2 \pm 1.4 \mu \mathrm{m})$ nanotubes are indistinguishable and comparable to the expected value for nanotubes 12 or 14 double helices ( 6 or 7 tiles) in circumference. ${ }^{3}$

These persistence lengths are several times longer than those observed previously. ${ }^{3}$ Here we used higher DNA concentrations and a slower thermal anneal, both of which should reduce the occurrence of lattice defects and favor larger tube diameters. The observation that ligation does not noticeably influence stiffness at room temperature reinforces the notion that lattice structure, rather than sticky end stability, is the primary determinant of nanotube stiffness.

With a persistence length of tens of micrometers, stiffness is not a major practical limitation. More limiting would be sensitivity to changes in solution conditions. In particular, high salt conditions are not compatible with many chemical reactions. Charge-stabilized colloids that one might wish to attach to the nanotubes, including many types of semiconductor quantum dots, aggregate in the presence of excess $\mathrm{Mg}^{2+}$. Buffer exchange may therefore be required for a number of chemistries aimed at functionalizing DNA nanotubes.

We sought to remove all potentially undesirable components from the nanotube buffer by exchanging the annealing buffer for pure water. We experimented with three methods of buffer exchange: dialysis, spin filtration, and ultracentrifugation (see the Supporting Information). Unligated nanotubes survive all three manipulations when solution conditions are kept constant, but fall apart immediately and completely when the buffer is exchanged for pure water, regardless of the method used. We therefore conclude that it is the change in solution composition rather than the mechanical manipulation involved in buffer exchange that destroys unligated DNA nanotubes. Four-corner ligated nanotubes survive all three buffer exchange procedures and are stable in pure water for at least one month.

Here we performed ligation after self-assembly to fortify a tile-based DNA lattice. Ligation may also be of use during self-assembly. For example, ligation restricts the torsional flexibility of nicked dsDNA. ${ }^{26}$ If a sticky end junction were ligated immediately following hybridization, it might usefully constrain the global geometry of a growing tile lattice. The relative kinetics of lattice formation and ligation will be crucial in determining the effects ligase has during selfassembly. The challenge in such an approach will be in avoiding ligations of improper hybridizations that could inhibit assembly of the desired structure.

In summary, we have shown that T4 DNA ligase seals nicks between tiles in DNA nanotubes, making them more robust to practical manipulations. The extent of ligation at individual corner nicks ranged from 46 to $85 \%$ with an average of $68-77 \%$ when three of the four corner nicks were $5^{\prime}$ phosphorylated. These results suggest that tile-based DNA superstructures are generally viable substrates for efficient ligation. Similar experiments on other tile-based lattices would help determine the geometrical constraints important for ligation and establish design principles for more fully ligatable DNA lattices. Ligation already plays a critical role in characterizing DNA nanostructures. In the future, it may be even more important as a means of fortifying these structures for technological applications.

Acknowledgment. We thank Erik Winfree and Kyle Chipman for insightful discussions. This work was supported by an NER Award No. 0404440 from the National Science Foundation.

Supporting Information Available: Gel preparation, band analysis, fluorescence microscopy, AFM, and persistence length measurements. This material is available free of charge via the Internet at http://pubs.acs.org.

\section{References}

(1) Liu, D.; Park, S. H.; Reif, J. H.; LaBean, T. H. Proc. Natl. Acad. Sci. U.S.A. 2004, 101, 717.

(2) Mitchell, J. C.; Harris, J. R.; Malo, J.; Bath, J.; Turberfield, A. J. J. Am. Chem. Soc. 2004, 126, 16342.

(3) Rothemund, P. W.; Ekani-Nkodo, A.; Papadakis, N.; Kumar, A.; Fygenson, D. K.; Winfree, E. J. Am. Chem. Soc. 2004, 126, 16344

(4) Braun, E.; Keren, K. Adv. Phys. 2004, 53, 441.

(5) Liu, Y.; Lin, C. X.; Li, H. Y.; Yan, H. Angew. Chem., Int. Ed. 2005 , $44,4333$.

(6) Yan, H.; Park, S. H.; Finkelstein, G.; Reif, J. H.; LaBean, T. H. Science 2003, 301, 1882. 
(7) Sharma, J.; Chhabra, R.; Liu, Y.; Ke, Y. G.; Yan, H. Angew. Chem., Int. Ed. 2006, 45, 730.

(8) Le, J. D.; Pinto, Y.; Seeman, N. C.; Musier-Forsyth, K.; Taton, T. A.; Kiehl, R. A. Nano Lett. 2004, 4, 2343.

(9) Noji, H.; Yasuda, R.; Yoshida, M.; Kinosita, K. Nature 1997, 386, 299.

(10) Petrillo, M. L.; Newton, C. J.; Cunningham, R. P.; Ma, R. I.; Kallenbach, N. R.; Seeman, N. C. Biopolymers 1988, 27, 1337.

(11) Yang, X. P.; Wenzler, L. A.; Qi, J.; Li, X. J.; Seeman, N. C. J. Am. Chem. Soc. 1998, 120, 9779.

(12) LaBean, T. H.; Yan, H.; Kopatsch, J.; Liu, F. R.; Winfree, E.; Reif, J. H.; Seeman, N. C. J. Am. Chem. Soc. 2000, 122, 1848.

(13) Sa-Ardyen, P.; Vologodskii, A. V.; Seeman, N. C. Biophys. J. 2003, 84, 3829.

(14) Winfree, E.; Liu, F. R.; Wenzler, L. A.; Seeman, N. C. Nature 1998, 394, 539.

(15) Johnson, A.; O’Donnell, M. Curr. Biol. 2005, 15, R90.

(16) Fu, T. J.; Seeman, N. C. Biochemistry 1993, 32, 3211.

(17) We used combinations of chemically phosphorylated and unphosphorylated oligomers synthesized by Integrated DNA Technologies (Skokie, IL). http://www.idtdna.com.

(18) In contrast, other tile designs exist that yield long "reporter" strands upon ligation as in ref 10 .
(19) This commercially available buffer (Fisher T4 DNA ligation buffer) was used for convenience. ATP and DTT are not necessary at this stage and thermally degrade during the anneal.

(20) Defining the detection limit by the lightest band in the gel, the absence of a slow moving band corresponding to a cyclized no. 3 strand means the extent of ligation of the nick in the tile core is $<15 \%$.

(21) To make sure that strand no. 3 was $5^{\prime}$ phosphorylated, a control experiment was performed in which strand no. 3 and a short (16 base) strand were hybridized to strand no. 1. After exposure to ligase, there was a clearly visible band corresponding to the ligation of the $5^{\prime}$ end of strand no. 3 to the short strand.

(22) Tuma, R. S.; Beaudet, M. P.; Jin, X. K.; Jones, L. J.; Cheung, C. Y.; Yue, S.; Singer, V. L. Anal. Biochem. 1999, 268, 278.

(23) Secondary structures were determined using the Mfold web server with $0.1 \mathrm{M} \mathrm{NaCl}, 20^{\circ} \mathrm{C}$. http://www.bioinfo.rpi.edu/applications/ $\mathrm{mfold} / \mathrm{old} / \mathrm{dna}$.

(24) Zuker, M. Nucleic Acids Res. 2003, 31, 3406.

(25) Doi, M.; Edwards, S. F. The Theory of Polymer Dynamics; Clarendon Press: Oxford, 1986.

(26) Zhang, Y. L.; Crothers, D. M. Abstr. Pap Am. Chem. Soc. 2003, 225, U520.

NL0603505 